\title{
Thermo-sensitive bFGF-modified Hydrogel with Dental Pulp Stem Cells on Neuroinflammation of Spinal Cord Injury
}

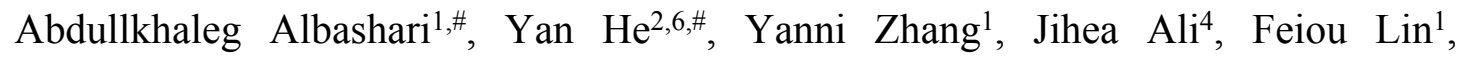
Zengming Zheng ${ }^{5}$, Keke Zhang ${ }^{1}$, Yanfan $\mathrm{Cao}^{1}$, Chun $\mathrm{Xu}^{6, *}$, Lihua Luo ${ }^{1, *}$, Jianming Wang ${ }^{2}$, Qingsong Ye $\mathrm{e}^{1,3,6, *}$

1 School and Hospital of Stomatology, Wenzhou Medical University, Wenzhou, Zhejiang, 325035, China

2 Laboratory for Regenerative Medicine, Tianyou Hospital, Wuhan University of Science and Technology, Wuhan, Hubei, 430064, China

${ }^{3}$ Centre of Regenerative Medicine, Renmin Hospital of Wuhan University, Wuhan University, Wuhan, Hubei, 430060, China

4 College of Life and Environmental Science, Wenzhou University, Wenzhou, Zhejiang, 325035, China

5 The Second Affiliated Hospital and Yuying Children's Hospital of Wenzhou Medical University, Wenzhou, Zhejiang, 325000, China

${ }^{6}$ School of Dentistry, The University of Queensland, 288 Herston Road, Brisbane, QLD 4006, Australia 


\section{Physi-chemical properties of the hydrogels}

As for the mechanical properties of the hydrogels, Figure S1.A displays the sol-gel transformation temperature of hydrogel between the groups. At low temperature (lower than $37^{\circ} \mathrm{C}$ ), both the loss and storage moduli of HeP showed small magnitude which increases drastically when increasing temperature range compare to PF127 due to the hydrogels forming phase. Figure S1.B presents the storage and loss moduli of angular frequency for $17 \mathrm{wt} \% \mathrm{PF} 127$ and $\mathrm{HeP}$ at $37{ }^{\circ} \mathrm{C}$. It shows that the storage modules of $\mathrm{HeP}$ are higher than the loss modules over a whole range of angular frequencies researched, significance that the linear viscoelastic characters of HeP system were controlled by an elastic nature. the data illustrated that the strain rate dependency of the shear stress for $17 \mathrm{wt} \% \mathrm{PF} 127$ and $\mathrm{HeP}$ at $37{ }^{\circ} \mathrm{C}$ (Figure S1.C). The shear stress of HeP leans toward level off and nears a limiting regular value as a decrease in strain rates toward zero at the low range of strain rates. From the Figure S1, it clearly appears that the HeP storage modulus displayed a linear character within a small range of strain amplitudes $(\gamma 0<1 \%)$ and then demonstrated a sharp decrease as a rise in strain amplitude. Furthermore, the results show that the storage modulus of HeP within a linear viscoelastic region slightly increases as an increase in angular frequency. 

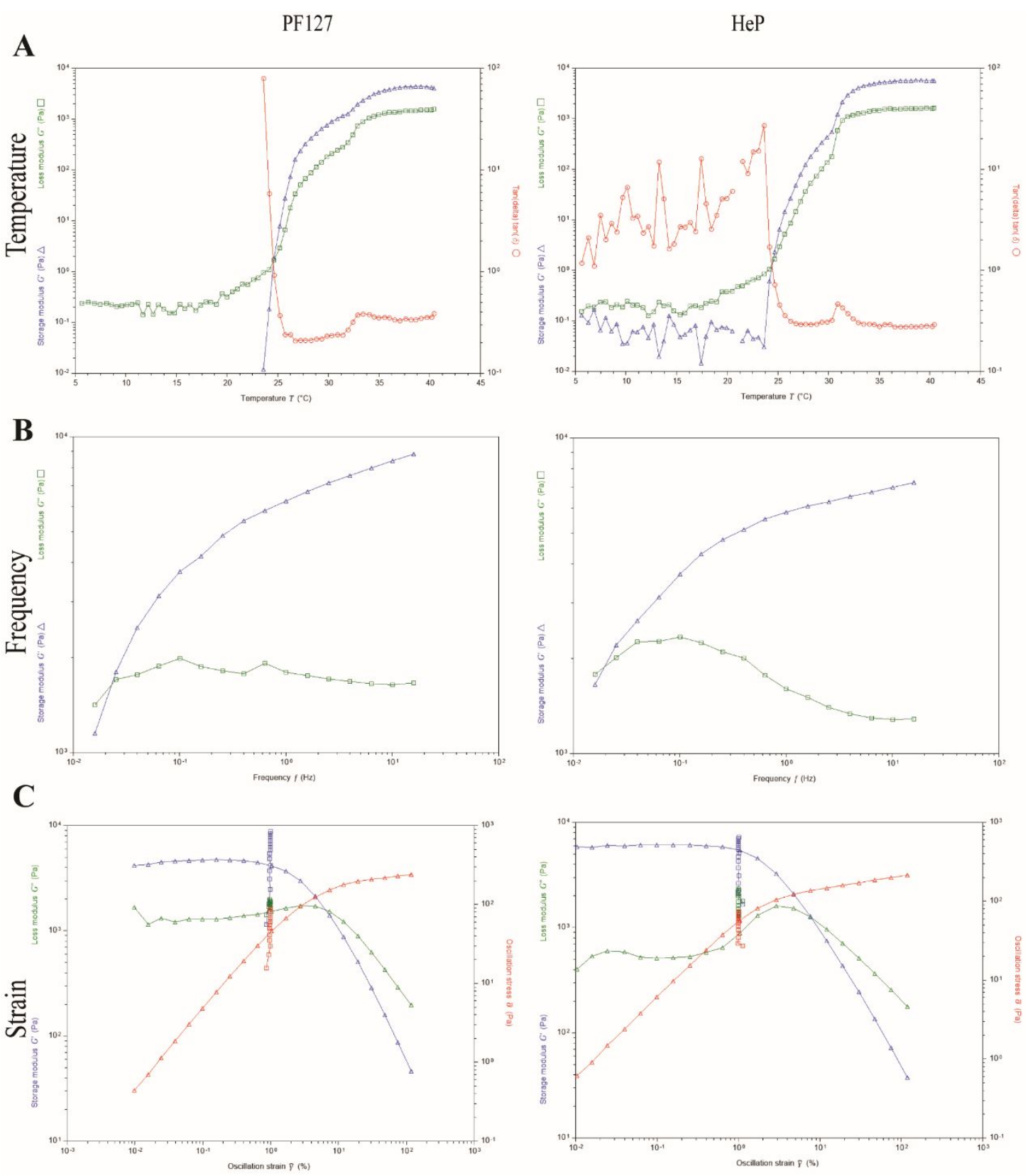

Figure S1. Physi-chemical characterization of hydrogels. 